\title{
A global envelope test to detect early and late bursts of trait evolution.
}

\section{J. Murrell ${ }^{1,2, *}$}

${ }^{1}$ Department of Genetics, Evolution and Environment, University College London, Gower Street, London WC1E 6BT, UK.

${ }^{2}$ Centre for Biodiversity and Environment Research, University College London, Gower Street, London WC1E 6BT, UK.

*Corresponding author: d.murrell@ucl.ac.uk

Running title:

Key-words: Disparity through time; adaptive radiation; phenotypic diversification; null model; morphological diversification; macroevolution; comparative methods 


\section{Abstract}

2 The joint analysis of species' evolutionary relatedness and their morphological evolution has

3 offered much promise in understanding the processes that underpin the generation of biological

4 diversity. Disparity through time (DTT) is a popular method that estimates the relative trait

5 disparity within and between subclades at each time point, and compares this to the null hypothesis

6 that trait values follow an uncorrelated random walk along the time calibrated phylogenetic tree. A

7 simulation envelope is normally created by calculating, at every time point, the $95 \%$ minimum and

$895 \%$ maximum disparity values from multiple simulations of the null model on the phylogenetic

9 tree. The null hypothesis is rejected whenever the empirical DTT curve falls outside of this

envelope, and these time periods may then be linked to events that may have sparked non-random

trait evolution. However, this method of envelope construction leads to multiple testing and a poor,

12 uncontrolled, false positive rate. As a consequence it cannot be recommended. A recently

13 developed method in spatial statistics is introduced that constructs a confidence envelope by giving

14 each DTT curve a single ranking value based upon its most extreme disparity value. This method

15 avoids the pitfalls of multiple testing whilst retaining a visual interpretation. Results using

16 simulated data show this new test has desirable type 1 properties and is at least as powerful in

17 correctly rejecting the null hypothesis as the morphological disparity index and node height test that

18 lack a visual interpretation. Three example datasets are reanalyzed to show how the new test may

19 lead to different inferences being drawn. Overall the results suggest the new rank envelope test

20 should be used in null model testing for DTT analyses, and that there is no need to combine the

21 envelope test with other tests such as has been done previously. Moreover, the rank envelope

22 method can easily be adopted into recently developed posterior predictive simulation methods.

23 More generally, the rank envelope test should be adopted when-ever a null model produces a vector

24 of correlated values and the user wants to determine where the empirical data is different to the null

25 model. 


\section{Introduction}

Understanding the joint temporal dynamics of taxonomic and phenotypic diversity can provide

tremendous insights into evolutionary success and its relationship with ecological opportunity,

selective pressures, constraints, biotic interactions and environmental conditions. At the most basic

level evolutionary biologists are often interested in detecting non-random evolution of biological

traits within and across clades of species. Non-random bursts in evolution are often thought to be

associated with events that open up ecological opportunities and enable a rapid increase in

such bursts in trait evolution may occur at other times and can be triggered by other processes such as major events in the external environment.

A variety of methods exist to look for the signature of evolutionary bursts and fall into the categories of null model testing, and model selection (Harmon et al., 2003, Freckleton and Harvey, 2006, Harmon et al., 2010, Slater et al., 2010, Slater and Pennell, 2014). The model selection approach takes a variety of candidate models (Brownian evolution, early burst, selective peak) and fits these to the data using maximum-likelihood methods before choosing the model that has the 'best fit' (Harmon et al., 2010, Slater and Pennell, 2014). The null model approach remains more popular, partly because the methods have been established for longer, and the overall aim is to investigate if the data can be distinguished from the null model of uncorrelated evolution of trait

One of the more popular null model approaches is to look at morphological traits to see if trait disparity increases, decreases or stays the same as species accumulate in evolutionary time, and also 53 see whether this disparity is greater within clades or between clades. Convergent evolution of traits 54 is implied if morphological disparity is predominantly found within one or more subclades; whereas 
55

56

adaptive radiations are expected to show divergence of traits between subclades, and in this scenario between clade morphological disparity should be greater than among subclade disparity.

This analysis of between and within clade trait disparity has been championed by the disparity through time (DTT) approach introduced by Harmon et al. (2003). Here the empirical DTT curve is compared to the distribution of DTT curves generated on the same phylogenetic tree but under a specific model of how the trait diversity evolves. Generally the null model is an uncorrelated random walk, and this is generally referred to as Brownian evolution (ie a Brownian random walk over time in trait space). The method of comparison is critical in determining whether the empirical data can be distinguished from the null model. Early analyses used an integral deviation method called the Morphological Disparity Index (MDI) which sums the deviations of the empirical DTT curve from the median of the null model simulations (Harmon et al., 2003). The index can then be compared to the distribution of values produced by the simulation to test whether it is significantly different from the null model (e.g. Ingram (2015)). Where MDI > 0, this implies within-clade trait variation is generally greater than expected under the null model, and MDI $<0$ implies betweenclade trait variation is more dominant than expected under the null model, and is suggestive of an adaptive radiation. The strength of the MDI is that it is a global test and so avoids multiple testing that can occur in analyses of time series data (see below), but the power of the MDI to detect nonBrownian bursts in trait evolution may be compromised if short-lived but extreme deviations in one direction at one point in the time series are balanced by a weak but long-lived deviation in the opposite direction.

Since the MDI produces a number, visualization of where any non-random bursts might have occurred (e.g. early on in the radiation) can only proceed by plotting the empirical DTT curve against the DTT curves sampled from the null model. However, determining where statistically significant local deviations from the null model are occurring in the time series requires another test. Slater et al. (2010) provided this by introducing an envelope method where the (100- $\alpha) \%$ 
81 upper and lower confidence intervals for the null model are estimated by sampling from the null

82 model $n$ times (where typically, $n>1000$ ) and then ordering the relative disparity values at each

83 time point to arrive at an envelope that contains $(100-2 \alpha) \%$ of the simulated relative disparity

values at each time point. This method is also referred to as the pointwise envelope method

(Myllymaki et al., 2017). The observed relative disparity can then be compared to this envelope and

if it falls outside the null model is said to be rejected at that level of significance.

The pointwise envelope method continues to be a popular method of inference. For example, Weber

et al. (2016) used the DTT method to investigate the macroevolution of perfume signalling in

orchid bees, a group known for their chemical sexual communications, and therefore a likely

candidate for rapid diversification in traits. They found strong non-Brownian evolution for perfume

signal, and weaker support for non-Brownian evolution of the labial gland. In both cases disparity

was greater than the median DTT of the null model simulations indicating clades are overlapping in

trait space, a signature of convergent evolution. The visual/graphical interpretation of the DTT with

an envelope test has extra appeal as it can be used to identify time points where the burst of non-

96 Brownian evolution occurred, enabling correlation with known evolutionary or environmental

events that have triggered the burst. For example, Aristide et al. (2016) investigated brain shape,

supported the conclusion that a burst in evolution of brain shape occurred approximately 17-12 Ma,

100 was associated with a burst in evolution of body mass that has previously been linked to

101 diversification of diet and locomotion strategies, and was followed by a slowdown in disparity

102 changes that persists to the current day. Conversely, Feilich (2016) found disparity in cichlid fin and

103 body morphology was often greater than expected under the null model of Brownian evolution

104 indicating most variation in morphology occurred within subclades. Moreover, the observed body

105 and median fin disparity above the $95 \%$ confidence interval produced by the null model simulations 
106 coincided with the Cichlinae-Pseudocrenilabrinae split, and a later split caused by the radiation of

107 the haplochromine cichlids.

108

109 However, the pointwise envelope method leads to weaker than expected statistical performance

110 because multiple tests, one at each time point, are being performed simultaneously. This is an issue

111 that occurs in many different areas (eg spatial statistics Baddeley et al. (2014)), and generally

112 whenever the pointwise envelope method is used in conjunction with a non-parametric method that

113 produces a function as its summary output. Multiple testing leads to an increased type 1 statistical

114 error rate (an elevated rate of rejection of the null hypothesis when it is true) that is no longer in line

115 with the significance level being used to generate the confidence intervals of the envelope.

116 Although multiple testing problems may be solved using a Bonferroni correction, it is not

117 appropriate here because the assumption of independence of tests is violated by the correlation of

118 disparity values between consecutive time points and also the (often) large number of time points

119 being simultaneously evaluated (Loosmore and Ford, 2006). Perhaps as a consequence of this many

120 studies, including those discussed above, have used multiple methods to look for non-Brownian

121 trait evolution including the MDI and the node height test (Freckleton and Harvey, 2006). However,

122 the continued use of the pointwise envelope suggests its graphical interpretation is very appealing

123 and it would therefore be worthwhile to circumvent its multiple testing issues.

125 Recently, a new method that avoids the multiple testing problems of the pointwise envelope but

126 retains the visual interpretation has been developed in spatial statistics (Myllymaki et al., 2017).

127 Spatial analysis of ecological data often leads to use of a non-parametric summary statistic such as

128 Ripley's K that plots the tendency to cluster against the radial distance (eg. Law et al. (2009)), and

129 the problem of pointwise envelopes for inference of non-random patterns is well established

130 (Loosmore and Ford, 2006, Baddeley et al., 2014). Instead of producing confidence intervals by

131 ranking each curve at each time point the rank envelope method ranks each curve for its overall 
132 extremness (more details are given below). As shown by Myllymaki et al. (2017), it has good type 1

133 and type 2 error rates and is recommended for testing point pattern data against the null model of

134 complete spatial randomness. The rank envelope can be developed and applied to any model that

135 produces a vector (eg van Veen and Murrell, 2005), however, its performance needs to be tested

136 since there are many ways of ordering curves based on their 'extremness' and not all methods will

137 produce desirable results.

In what follows, the rank envelope test will be developed for DTT null model analyses and its type

1401 and type 2 statistical properties compared to the pointwise envelope, MDI, and node height tests.

141 The pointwise envelope test will be shown to have extremely poor type 1 error rates and should not

142 be used for inference. In contrast, the rank envelope method will be shown to possess desirable type

1431 error rates, and be at least as powerful as the other tests in detecting accelerating or decelerating

144 rates of trait evolution whilst retaining the useful property of graphical interpretation.

\section{Methods}

\section{Data simulation}

148 Phylogenetic trees were generated within $R$ (version 3.3.3) using the pbtree function with the

149 phytools R library (version 0.6, Revell (2012)), using the pure-birth (Yule) model. A pure-birth

150 model is a simple way to ensure the number of tips is constant across simulations, but may be

151 biologically plausible for clades over relatively short periods of time. These phylogenetic trees were

152 then used to simulate quantitative trait evolution under a variety of scenarios including the null

153 model of Brownian evolution. Specifically, trait evolution was simulated using the fastBM (in

154 phytools) and rescale (in geiger, version 2.0.6, Pennell et al. (2014)) functions. The rescale function

155 allows the simulation of early or late burst trait evolution using the early burst option, and a rate

156 change parameter, $a$. When $a<0$ the rate of evolution decreases with time, mimicking an early

157 burst of trait evolution, whereas $a>0$ models a late burst. The magnitude of $a$ determines how 
158 quickly this burst of activity fades away or builds up, with large magnitudes delivering a rapid

159 decay or late increase in evolutionary change (examples are given in Figure S1 of the

160 supplementary information). The null model of Brownian evolution is simulated under the same

161 framework, but where $a=0$.

162

163

Disparity Through Time (DTT) Analyses

164 DTT has proven to be one of the more popular approaches and uses the average pairwise Euclidean

165 distance between species trait values as a measure of disparity. Following Harmon et al. (2003)

166 relative disparity is calculated by dividing disparity of each subclade by the disparity of the whole

167 tree. At each time point (speciation event) the average relative disparity for that time point is

168 calculated as the mean of the relative disparities for all subclades whose ancestral lineages are

169 present at that time. Disparity values close to zero indicate that variation in the trait(s) is

170 predominantly partitioned between subclades rather than within them. Disparity values close to

171 unity suggest that a clade contains a large amount of that variation, and that clades may overlap in

172 trait space. By definition, disparity is 1 at the base of the phylogenetic tree, but is 0 at the present

173 day.

174

175 Two methods that are currently used to search for the signal of bursts in morphological evolution

176 using DTT are (1) the pointwise envelope test (Slater et al., 2010), and (2) an integral deviation test

177 known as the Morphological Disparity Index (MDI, Harmon et al. (2003)). As well as these a third

178 test, the global envelope test, was investigated. The global envelope test retains the same visual

179 interpretation as the pointwise envelope test whilst avoiding the problem of multiple testing. All

180 make comparisons of the empirical DTT to the DTT taken from the ensemble of simulations

181 generated by the null model of Brownian evolution, and all use the same measure of disparity

182 defined above.

183 


\section{The Pointwise Envelope Test}

185 The pointwise envelope test is a Monte-Carlo simulation method that aims to produce a confidence

186 interval, or envelope within which any part of the empirical DTT curve is said to be statistically

187 indistinguishable from the null model. The method currently implemented in Geiger (v2.0.6 )

188 constructs an envelope by extracting the $\alpha$ th and (100- $\alpha)$ th quantile of the DTT at each time point

189 (speciation event) for all the null model simulations (normally $\alpha=2.5$ ). This produces a lower and

190 upper interval within which $(100-2 \alpha) \%$ of all values for DTT at each speciation event under the null

191 model. More formally, the envelope is defined by the lower and upper bounding curves

$$
T_{l o w}^{(k)}(t)=\min _{i=1,2, \ldots . s}^{k} T_{i}(t)
$$

$T_{u p p}^{(k)}(t)=\max _{i=1,2, \ldots . s}^{k} T_{i}(t)$

194 where $\min ^{k}$ and $\max ^{k}$ denotes the $k$ th smallest and largest values of the DTT across all simulations

$195 s$, of the null model at time (speciation event) $t$. If the empirical DTT curve falls outside of this

196 envelope it is interpreted as being evidence for a departure from the null model of Brownian

197 evolution.

\section{The MDI test}

200 Perhaps the simplest way to avoid multiple testing is to perform a deviation test that sums the

201 deviations of the empirical DTT from the median DTT of the ensemble of null model simulations.

202 Known as the Morphological Disparity Index (MDI), negative values indicate the empirical DTT

203 curve is below the null model median DTT for at least some of the range of time points, again

204 pointing to the possibility of an early burst in diversity (Harmon et al., 2003). A disadvantage of

205 this approach is that it is not possible to say where the empirical DTT deviates from the null model

206 without plotting it against the null model simulations and then performing some sort of envelope

207 test. Moreover, since the index sums up the deviations from the median of the ensemble of

208 simulations of the null model, it is theoretically possible for time periods where the empirical DTT

209 is above the median DTT to be cancelled out by time periods where it is below the median DTT, 
210 thus giving an MDI value close to that expected under Brownian evolution. However, most likely

211 because it avoids the issue of multiple testing, the MDI has been well used (eg Harmon et al.

212 (2003), Slater et al. (2010), Colombo et al. (2015), Ingram (2015), Jonsson et al. (2015)). In the

213 results below Monte Carlo simulations were used to generate a distribution of MDI values from the

214 null model. The empirical MDI value is then compared to this distribution and the null hypothesis

215 (that the empirical data is from the same distribution as the null model of Brownian evolution) is

216 rejected if it is smaller than the $\alpha$ th quantile value or larger than the $(100-\alpha)$ th quantile. As for the

217 envelope tests, $\alpha=2.5$.

Rank Envelope Test

220 A method that avoids multiple testing and therefore one which should show better type 1 error 221 properties is the rank envelope test. In this case the whole DTT curve is ranked in terms of its 222 extremness (rather than rank each speciation event individually). This so-called 'extreme rank' 223 depth measure method was recently introduced by Myllymaki et al. (2017) for a similar problem 224 that occurs in hypothesis testing for spatial point processes where non-parametric functions (pair 225 correlation function and Ripley's K) are used to detect departures from complete spatial 226 randomness (ie a lack of any correlation between points). Such tests have been popular in ecology 227 (e.g. Wiegand and Moloney (2004), Flügge et al. (2012)). The more formal underpinnings of the 228 test can be found in Myllymaki et al. (2017), but briefly the DTT curves from the $r$ simulations of 229 the null model are ordered according to the largest $k$ for which they are still present in the $k$ th 230 envelope as defined by the pointwise envelope (equations 1a and $1 \mathrm{~b}$ ).

$$
R_{i}=\max \left\{k: T_{\text {low }}^{(k)}(t) \leq T_{i}(t) \leq T_{u p p}^{(k)}(t) \text { for all } t\right\}
$$
ranking within the pointwise envelope. 
236 Since ties are possible (indeed are likely) the set of curves can only be weakly ordered. In order to

237 generate a $p$-value a method for dealing with the ties needs to be used. Following Myllymaki et al.

238 (2017), a range of $p$-values is reported that encompasses the most liberal and most conservative $p$ -

239 values respectively defined as

$$
\begin{aligned}
& p_{-}=\frac{1}{s+1} \sum_{i=1}^{s+1} 1\left(R_{i}<R_{1}\right) \\
& p_{+}=\frac{1}{s+1} \sum_{i=1}^{s+1} 1\left(R_{i} \leq R_{1}\right)
\end{aligned}
$$

and where $R_{1}$ is the rank of the empirical DTT curve. This does raise the further problem that the

243 interval defined by $p_{-}$and $p_{+}$could include the significance level $\alpha$, leading to an ambiguous result.

244 However the likelihood of this happening is very small as long as $s$, the number of Monte Carlo

245 simulations of the null model is sufficiently large. Myllymaki et al. (2017) recommend $s \geq 2500$,

246 and the results below use $s=5000$. From the above DTT curve ordering, it is straightforward to

247 visualise the global envelope determined by the significance level used, and in so-doing the user

248 can readily see where the empirical data falls outside of the global envelope, and thus where the

249 DTT is significantly different to Brownian evolution.

251 The extreme depth ranking method used here is but one of a number of possible ways to order the

252 DTT curves. Some other functional depth orderings are discussed in Myllymaki et al. (2017) and

253 the reader is directed to their paper for more details, but as will be shown below, the extreme rank

254 depth measure described above leads to desirable type 1 and type 2 statistical errors for DTT

255 analyses.

\section{The Node Height Test}

258 The final test does not use simulations of the null model to compare to the empirical data but 259 instead relies upon the expectation that trait evolution should slow as niche space become packed.

260 The node height test (Freckleton and Harvey, 2006) investigates if there is a significant correlation

261 between the absolute magnitude of the standardized independent contrasts of the trait(s) and the 
262 height above the root of the node at which they were being compared to. The height of a node is

263 defined as the absolute distance between the root and the most recent common ancestor of the pair

264 from which the contrast is generated. A significant relationship between these indicates that the rate

265 of trait evolution is changing systematically through the tree with early and late bursts in trait

266 evolution being diagnosed by the sign of the slope. Since this is an established test, the analyses of

267 the node height test were performed using the function nh.test with the R library geiger (version

268 2.0.6).

Software code

271 All results were obtained using $R$ (version) and the code used to produce the results reported below

272 can be found at https://github.com/djmurrell/DTT-Envelope-code. Details of the current methods to

273 detect non-Brownian bursts of trait evolution can be found in the geiger software library, and the

274 rank envelopes are produced by modifying code from the spptest library (Myllymaki et al., 2017).

\section{Results}

277 False positive rates (Type 1 errors)

278 The false positive rate is investigated by simulating an empirical dataset of trait evolution under the

279 Brownian null model and testing how frequently each of the four tests described above incorrectly

280 rejects the null hypothesis. Results for the DTT approach using the pointwise envelope test at the

$2815 \%$ level of significance show a disappointing, but unsurprising high rate of false positives (Figure

282 1). The multiple testing nature of this method means that the false positive rate is dependent upon

283 the number of species in the comparison, and in the simulations the rate of false positives ranges

284 approximately between 0.25 and 0.5 for $10-200$ species (Figure 1 ). That is to say, for comparisons

285 using more than 100 species the pointwise envelope test is incorrectly rejecting the null hypothesis

286 of Brownian evolution in approximately $50 \%$ of cases. As such it is impossible to recommend this

287 method for inference of non-Brownian bursts of trait evolution. In comparison, the node height test 
and the global rank envelope test both return consistent false positive rates that hover around the

significance level used (Figure 1). The MDI shows a high type 1 error rate for phylogenetic trees

with less than 40 species, but thereafter a desirable false positive rate is returned.

293 Evolutionary biologists are often most interested in early bursts in trait evolution, since this is

294 argued to be the hallmark of adaptive radiations (Harmon et al., 2010). However, a number of

295 studies have also reported late bursts in trait evolution where departures from Brownian evolution

296 occur only in the recent past (e.g. Koecke et al. (2013), Tran (2014), Pincheira-Donoso et al. (2015),

297 Feilich (2016)). Simulations for both scenarios confirm that the MDI, the node height, and the rank

298 envelope test can all successfully detect both early and late bursts in trait evolution (Figure 2).

299 Convention dictates that at the 5\% significance level a desirable test shows a true positive rate of

300 0.8. The ability of all tests to reach this mark is dependent on the number of species and the strength

301 of the early or late burst, but other generalities do emerge. Firstly, for early burst trait evolution the

302 MDI deviation test is less powerful than the node height and global envelope tests, with the global

303 envelope test generally showing slightly higher power. Secondly, early bursts are slightly easier to

304 detect than late bursts of trait evolution i.e. the desired true positive rate is reached with fewer

305 species in early burst models compared to late burst models. The node height and global envelope

306 tests show similar power to detect late bursts, but the MDI is better able to detect late bursts in trait

307 evolution with fewer species at the tips. Example simulations for each early/late burst rate with 100

308 species at the tips of the phylogenetic tree can be found in the supplementary material (Figure S1).

310 Data Examples

311 Having established the rank envelope test possesses desirable type 1 and type 2 statistical error

312 properties, three datasets were used to illustrate how inference of the rates of morphological

313 evolution can change depending on whether the pointwise or global envelope test is used. Since the 
314 pointwise envelope test is too liberal in its rejection of the null model the expectation should be for

315 a reduction in support for non-Brownian bursts in morphological evolution.

317 The first example uses the morphological and phylogenetic data on Darwin's finches (Geospiza)

318 which is currently found in the geiger (version 2.0.6) $R$ package. Re-analysis shows support for two

319 late bursts in culmen length evolution by the pointwise envelope test, both showing diversification

320 predominantly occurring within clade(s) followed by decreases in disparity caused by increased

321 diversity between clades (Figure 3a). In contrast, there is no departure from the null model of

322 Brownian evolution according to the rank envelope test (Figure 3b).

324 The second example uses a time-calibrated molecular phylogeny of extant cetaceans and a

325 morphological dataset on body size from Slater et al. (2010) which is also located within geiger

326 (version 2.0.6). Their analyses used a combination of the node height test (NHT), and DTT tests

327 using the MDI and the DTT pointwise envelope method. On the basis of the pointwise envelope

328 results they came to the conclusion that cetaceans do show a burst in evolution of body size and that

329 this occurred predominantly during the period 6-11 Ma. Reanalysis confirms that the pointwise

330 envelope approach finds the same burst in trait evolution but that the global envelope test fails to

331 find any departure from the null model of Brownian evolution at the $5 \%$ level of statistical

332 significance (Figure 3c, d). These results are covered in more detail in the discussion.

334 The final example is taken from (Feilich, 2016) who investigated the evolution of

335 body shape, caudal fin shape, dorsal fin shape, and anal fin shape in 131 African cichlid fishes. Re-

336 analysing the data for anal fin shape using the pointwise envelope (Figure 3e) confirms the spike in

337 relative disparity coinciding with the Cichlinae-Pseudocrenilabrinae split 45-75 MYA reported in

338 the original paper as well as the spike nearer to the present day that coincides with the

339 haplochromine radiation (Feilich, 2016). In contrast, the rank envelope method finds no discernable 
difference (at the 5\% level of significance) from the null model of Brownian evolution at any point

341 in the evolutionary timeline (Figure 3f). Re-analysis of body shape, dorsal fin shape, and caudal fin

342 shape evolution using the rank envelope method does retain support for the peaks in disparity

343 associated with the haplochromine radiation found by (Feilich, 2016) using the pointwise envelope

344 method (Figure S2).

\section{Discussion}

347 The object of this study has been to highlight the unacceptably high false positive rates of the 348 pointwise envelope test, and offer an alternative solution, the global rank envelope test (Myllymaki 349 et al., 2017) that does not have the same multiple testing issues, but which still allows identification 350 of the time period where the non-Brownian trait evolution may have occurred. Envelope tests using 351 the DTT pointwise envelope method continue to be useful and popular (e.g. Johnson and Omland, 352 2004, Slater et al., 2010, Dornburg et al., 2011, Blackburn et al., 2013, Ingram, 2015, Arbour and 353 Lopez-Fernandez, 2016, Aristide et al., 2016, Feilich, 2016, Hlusko et al., 2016, Weber et al., 2016)

354 as the time periods over which trait evolution has been non-Brownian can often be linked to

355 specific events that may have triggered the burst of trait evolution (e.g. Slater et al., 2010, Feilich, 356 2016, Hlusko et al., 2016). Unfortunately, the results presented here (Figures 1- 3) suggest re-

357 interpretation of some previous analyses may be required using a global envelope test such as that 358 introduced here rather than a pointwise envelope test.

360 A number of methods beyond the pointwise envelope test have been used and there is clear 361 variability in their ability to detect departures from the null model (Figure 2). The MDI (Harmon et 362 al., 2003) is a global test as it sums up the difference between the empirical DTT and the average of 363 the simulations of the null model. However, the MDI can be relatively insensitive to early bursts in 364 diversity that lead to larger between-clade disparity (Figure 2); can return a high false positive rate 365 for datasets with less than 40 species (Figure 1); and it is possible for a large difference from the 
366 null model in one time period to be cancelled out by smaller deviations in the opposite direction at

367 other time periods. However, the MDI might be better at detecting late bursts in diversity (Figure

368 2). This is possibly because late bursts are often associated with long time periods of disparity

369 concentrated within clades (eg Figure 2a, e) and there is less chance the deviation from the median

370 of the simulations is cancelled out by negative deviations elsewhere. On the other hand, there is

371 little to choose between the rank envelope test and node height test based upon the results presented

372 here (Figure 1,2). The main advantage of the rank envelope method is that it provides a

373 visualization of how disparity changes over time and it is easier to see where the burst of trait

374 evolution may have occurred. Given this, and the slightly better performance when species numbers

375 are small and/or early/late bursts are weak, the rank envelope method might be preferable.

376

377 Tests using three datasets that are freely available show how inferences and conclusions can change

378 in quite important ways when the rank envelope test is used instead of the pointwise envelope

379 (Figure 3). Macroevolutionary investigations have often used multiple methods (eg node height test,

380 MDI test and DTT plots (Slater et al., 2010); MDI test and DTT plots (Arbour and Lopez-

381 Fernandez, 2016)), to test for departures from Brownian evolution, but given the results presented

382 here there is a risk that a mixture of results will be produced. For example, before removing species

383 considered to be outliers, Slater et al. (2010) found evidence supporting an early burst in cetacean

384 body size using the pointwise envelope test (replicated here in Figure 3), but neither the MDI test

385 nor the node height test could find a statistically significant deviation from the null model of

386 Brownian evolution. These discrepancies are expected given the high false positive rates of the

387 pointwise envelope test (Figure 1), and re-analysis using the global rank envelope test shows

388 agreement with the results of the initial node height test, and MDI test before removal of outliers

389 (Slater et al. (2010); Figure 3). 
391 The methods investigated here all use the same hypothesis testing approach. That is to say we test

392 our data against a suitable null model to see if there are detectable departures from the null model.

393 A different approach is to consider a number of candidate models and ask which model best

394 describes the data (Johnson and Omland, 2004). The advantage of this model selection approach is

395 that multiple models are considered simultaneously, but of course there is no guarantee that the best

396 model, usually determined by some information theoretic criterion, is a 'good' descriptor of the

397 data. The model selection approach has been developed for trait evolution by Harmon et al. (2010)

398 who used maximum likelihood methods to fit models that could produce Brownian evolution,

399 increasing or decreasing trait diversification rates, as well as selective peaks where the trait value

400 has a tendency to return to a medial value. Using the likelihood ratio test, they found the Brownian

401 evolution and the selective peak (Ornstein-Uhlenbek) models to be the most frequently selected

402 across 49 clades, implying early bursts in trait evolution are relatively rare. Slater and Pennell

403 (2014) extended this method by employing a posterior predictive approach instead of the likelihood

404 ratio test. The posterior predictive approach proceeds by fitting the parameters to the candidate

405 models using maximum likelihood as in (Harmon et al., 2010), but model selection is based upon

406 sampling the trait evolution from the fitted models and then comparing the fit of each model to the

407 observed trait values. Slater and Pennell (2014) developed this method using either the MDI test, or

408 the node height test and showed both of these posterior predictive methods can have a higher power

409 to detect early bursts in trait evolution compared to the maximum likelihood ratio approach of

410 Harmon et al. (2010). Re-analysing the cetacean dataset with these methods led to the conclusion

411 that an early burst model best described the evolution of whale body size (Slater and Pennell, 2014).

412 This is not surprising given the rank envelope test clearly shows the empirical DTT curve is close to

413 falling below the lower confidence interval (Figure 2d). Ultimately, the user needs to choose

414 between the null model testing and model selection methods, but the rank envelope test developed

415 here could easily be incorporated into the posterior predictive methods of Slater and Pennell (2014),

416 since the ranking of the observed DTT curve in the ensemble of simulations from each of the 
417 candidate models generates a single metric, the global rank amongst the set of model curves, that

418 could then be used to compare the models.

419

420 In summary, the pointwise envelope test method that has been employed to investigate bursts in 421 trait evolution shows unacceptably high type 1 statistical errors and should not be used. The rank 422 envelope test that was introduced by Myllymaki et al. (2017) for spatial point pattern analysis, ranks 423 the extremness of the empirical DTT curve against the suite of realisations of an appropriate null 424 model, and is instead shown to possess the desirable statistical properties. This method is shown to 425 be at least as powerful as other popular methods for detecting bursts of trait evolution, whilst 426 retaining the advantages of graphical interpretation of where in time the significant bursts of 427 evolution occur. Null model testing is a commonly used tool in ecology and evolution (eg Gotelli 428 and Ulrich (2012)) and the rank envelope test is a flexible method that allows inference without the 429 problem of multiple testing when the null model returns a functional relationship (ie a vector) rather 430 than a singular value (ie a scalar quantity).

\section{Acknowledgements}

434 This work was supported by the Engineering and Physical Sciences Research Council grant

435 EP/N007336/1. The author would like to thank Alex Pigot who provided useful suggestion and 436 comments on earlier versions of the manuscript. 


\section{References}

440 Arbour, J. H. \& Lopez-Fernandez, H. (2016) Continental cichlid radiations: functional diversity reveals the role of changing ecological opportunity in the Neotropics. Proceedings of the

Aristide, L., dos Reis, S. F., Machado, A. C., Lima, I., Lopes, R. T. \& Perez, S. I. (2016) Brain shape convergence in the adaptive radiation of New World monkeys. Proceedings of the National Academy of Sciences of the United States of America, 113, 2158-2163.

Baddeley, A., Diggle, P. J., Hardegen, A., Lawrence, T., Milne, R. K. \& Nair, G. (2014) On tests of spatial pattern based on simulation envelopes. Ecological Monographs, 84, 477-489.

Blackburn, D. C., Siler, C. D., Diesmos, A. C., McGuire, J. A., Cannatella, D. C. \& Brown, R. M. (2013) An adaptive radiation of frogs in a southeast Asian island archipelago. Evolution, 67, 2631-46.

Colombo, M., Damerau, M., Hanel, R., Salzburger, W. \& Matschiner, M. (2015) Diversity and disparity through time in the adaptive radiation of Antarctic notothenioid fishes. Journal of Evolutionary Biology, 28, 376-394.

Dornburg, A., Sidlauskas, B., Santini, F., Sorenson, L., Near, T. J. \& Alfaro, M. E. (2011) The influence of an innovative locomotor strategy on the phenotypic diversification of

Feilich, K. L. (2016) Correlated evolution of body and fin morphology in the cichlid fishes. Evolution, 70, 2247-2267.

Flügge, A. J., Olhede, S. C. \& Murrell, D. J. (2012) The memory of spatial patterns: changes in local abundance and aggregation in a tropical forest. Ecology, 93, 1540-1549.

Freckleton, R. P. \& Harvey, P. H. (2006) Detecting non-Brownian trait evolution in adaptive radiations. PLoS Biol, 4, e373. 180. 
465

466

467

468

469

470

471

472

473

474

475

476

477

478

479

480

481

482

483

484

Harmon, L. J., Losos, J. B., Davies, T. J., Gillespie, R. G., Gittleman, J. L., Jennings, W. B., Kozak, K. H., McPeek, M. A., Moreno-Roark, F., Near, T. J., Purvis, A., Ricklefs, R. E., Schluter, D., Schulte, J. A., Seehausen, O., Sidlauskas, B. L., Torres-Carvajal, O., Weir, J. T. \& Mooers, A. O. (2010) Early bursts of body size and shape evolution are rare in comparative data. Evolution, 64, 2385-2396.

Harmon, L. J., Schulte, J. A., 2nd, Larson, A. \& Losos, J. B. (2003) Tempo and mode of evolutionary radiation in iguanian lizards. Science, 301, 961-4.

Hlusko, L. J., Schmitt, C. A., Monson, T. A., Brasil, M. F. \& Mahaney, M. C. (2016) The integration of quantitative genetics, paleontology, and neontology reveals genetic underpinnings of primate dental evolution. Proceedings of the National Academy of Sciences of the United States of America, 113, 9262-9267.

Ingram, T. (2015) Diversification of body shape in Sebastes rockfishes ofthe north-east Pacific. Biological Journal of the Linnean Society, 116, 805-818.

Johnson, J. B. \& Omland, K. S. (2004) Model selection in ecology and evolution. Trends in Ecology \& Evolution, 19, 101-108.

Jonsson, K. A., Lessard, J. P. \& Ricklefs, R. E. (2015) The evolution of morphological diversity in continental assemblages of passerine birds. Evolution, 69, 879-889.

Koecke, A. V., Muellner-Riehl, A. N., Pennington, T. D., Schorr, G. \& Schnitzler, J. (2013) Niche evolution through time and across continents: The story of neotropical cedrela (Meliaceae) American Journal of Botany, 100, 1800-1810.

Law, R., Illian, J., Burslem, D. F., Gratzer, G., Gunatilleke, C. \& Gunatilleke, I. (2009) Ecological information from spatial patterns of plants: insights from point process theory. Journal of Ecology, 97, 616-628.

Loosmore, N. B. \& Ford, E. D. (2006) Statistical inference using the G or K point pattern spatial statistics. Ecology, 87, 1925-1931. 
Myllymaki, M., Mrkvicka, T., Grabarnik, P., Seijo, H. \& Hahn, U. (2017) Global envelope tests for spatial processes. Journal of the Royal Statistical Society Series B-Statistical Methodology, 79, 381-404.

Pennell, M. W., Eastman, J. M., Slater, G. J., Brown, J. W., Uyeda, J. C., FitzJohn, R. G., Alfaro, M. E. \& Harmon, L. J. (2014) geiger v2.0: an expanded suite of methods for fitting macroevolutionary models to phylogenetic trees. Bioinformatics, 30, 2216-2218.

Pincheira-Donoso, D., Harvey, L. P. \& Ruta, M. (2015) What defines an adaptive radiation? Macroevolutionary diversification dynamics of an exceptionally species-rich continental lizard radiation. Bmc Evolutionary Biology, 15.

Revell, L. J. (2012) phytools: an R package for phylogenetic comparative biology (and other things). Methods in Ecology and Evolution, 3, 217-223.

Schluter, D. (2000) The ecology of adaptive radiation. OUP Oxford.

Slater, G. J. \& Pennell, M. W. (2014) Robust regression and posterior predictive simulation increase power to detect early bursts of trait evolution. Syst Biol, 63, 293-308.

Slater, G. J., Price, S. A., Santini, F. \& Alfaro, M. E. (2010) Diversity versus disparity and the radiation of modern cetaceans. Proc Biol Sci, 277, 3097-104.

Tran, L. A. P. (2014) The role of ecological opportunity in shaping disparate diversification trajectories in a bicontinental primate radiation. Proceedings of the Royal Society BBiological Sciences, 281.

van Veen, F. \& Murrell, D. (2005) A simple explanation for universal scaling relations in food webs. Ecology, 86, 3258-3263.

Weber, M. G., Mitko, L., Eltz, T. \& Ramirez, S. R. (2016) Macroevolution of perfume signalling in orchid bees. Ecology Letters, 19, 1314-1323.

Wiegand, T. \& Moloney, K. (2004) Rings, circles, and null $\square$ models for point pattern analysis in ecology. Oikos, 104, 209-229. 


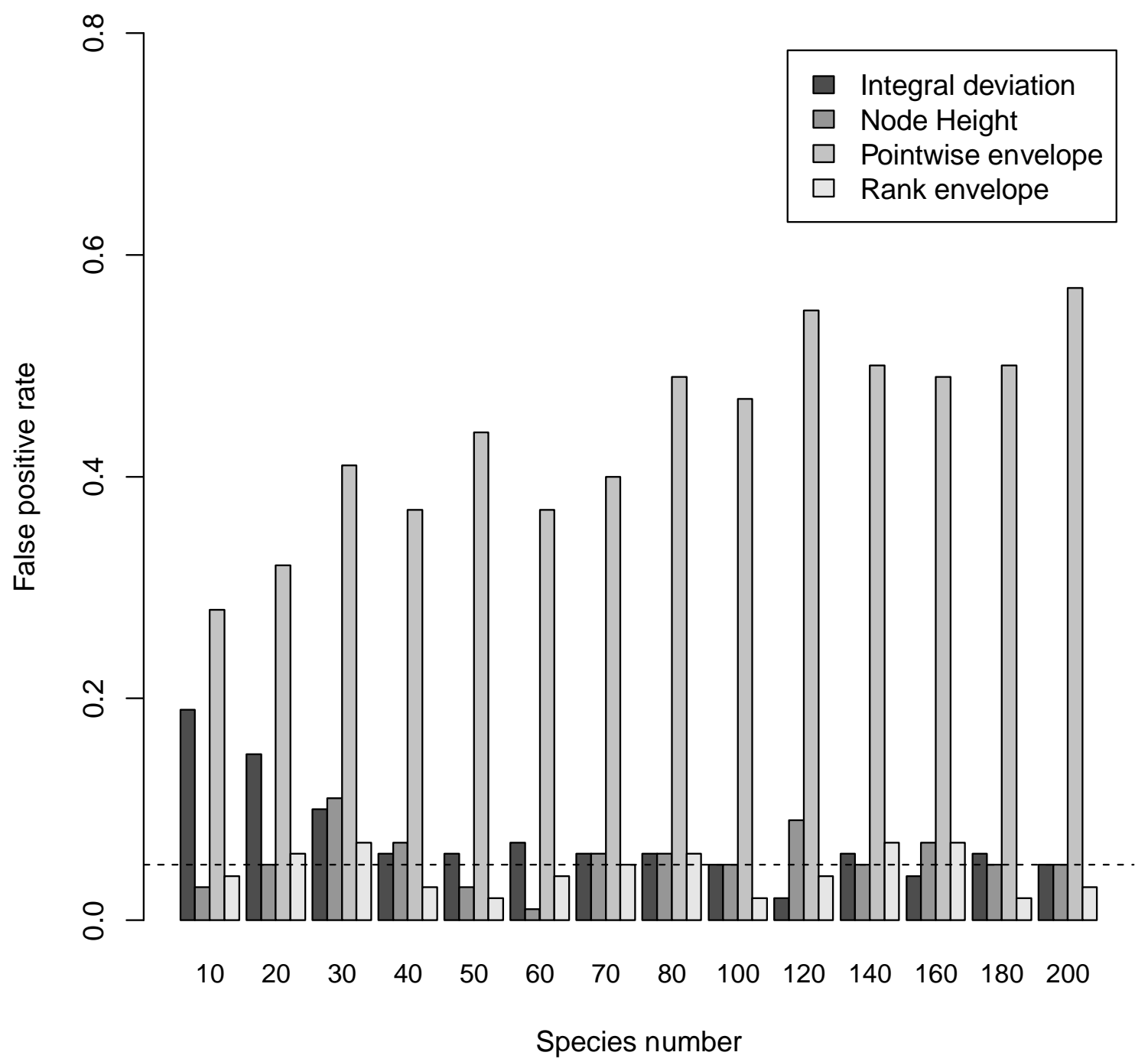

517 Figure 1. False positive rates for the four tests for non-random disparity through time (DTT) as a

518 function of the number of species at the tips of the phylogenetic tree. Rates are estimated from 100

519 simulated phylogenetic trees for each number of species using a pure birth model to generate the

520 phylogenetic tree, and assuming Brownian evolution of the trait at each speciation event. All tests

521 requiring Monte Carlo simulations were run with $s=5000$ trait evolution simulations. 
bioRxiv preprint doi: https://doi.org/10.1101/175968; this version posted September 5, 2017. The copyright holder for this preprint (which was not certified by peer review) is the author/funder, who has granted bioRxiv a license to display the preprint in perpetuity. It is made available under aCC-BY-NC-ND 4.0 International license.
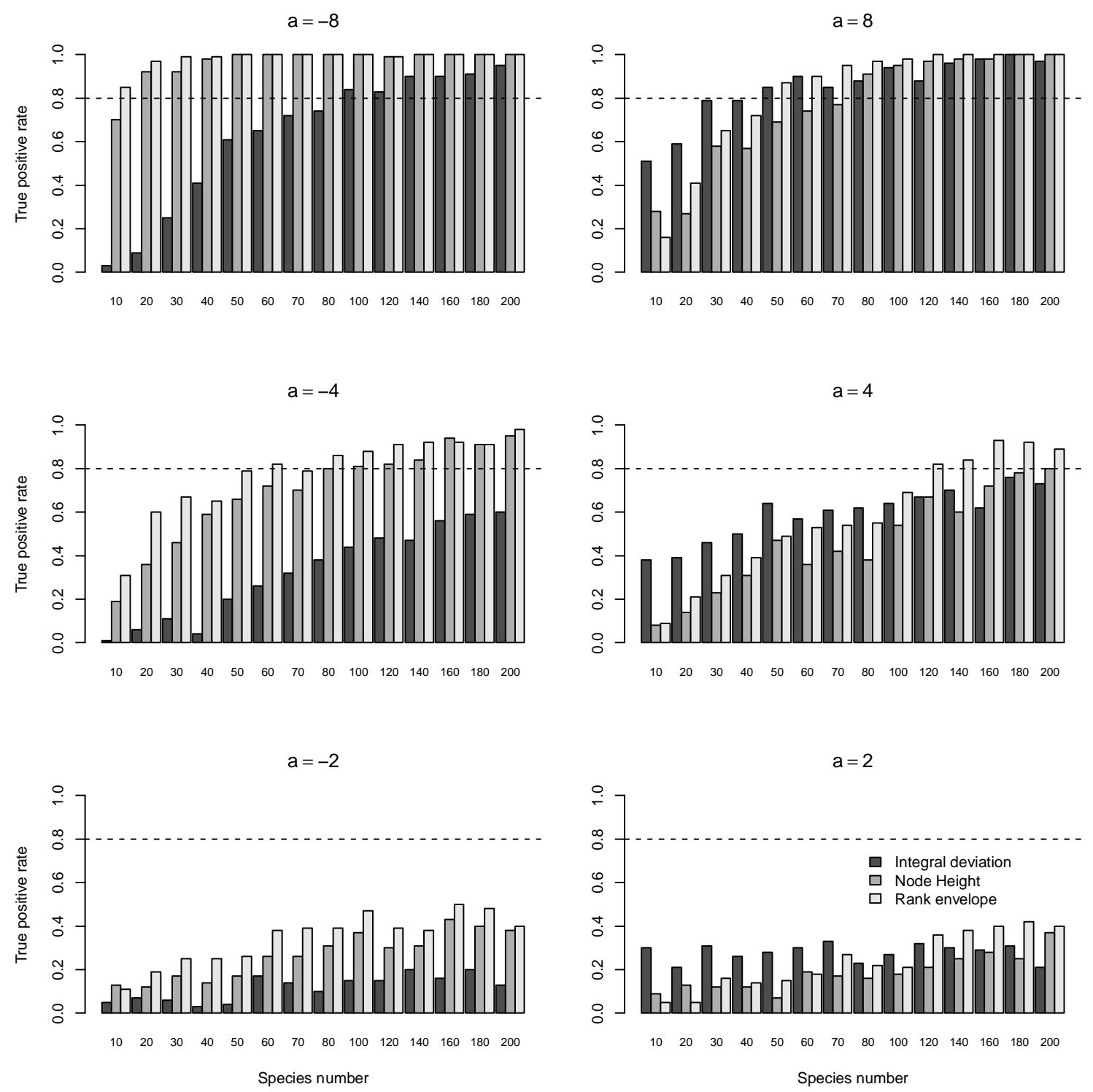
524 Figure 2. True positive rates (statistical power) of the rank envelope test, the MDI test and the node

525 height test under a range of simulated early and late burst scenarios, and for a range of size of

526 phylogenetic tree. Rates are estimated from 100 simulated phylogenetic trees for each number of

527 species using a pure birth model to generate the phylogenetic tree, and assuming trait evolution at

528 each speciation event speeds ups or slow downs over evolutionary time. All tests requiring Monte

529 Carlo simulations were run with $s=5000$ trait evolution simulations for every phylogenetic tree.

530 When $a<0$, a burst in trait evolution occurs early in evolutionary time, and late bursts occur when

$531 a>0$. Large magnitudes lead to the bursts occurring over a smaller period of time. Corresponding

532 example plots of DTT in each scenario are given in the supplementary information. 

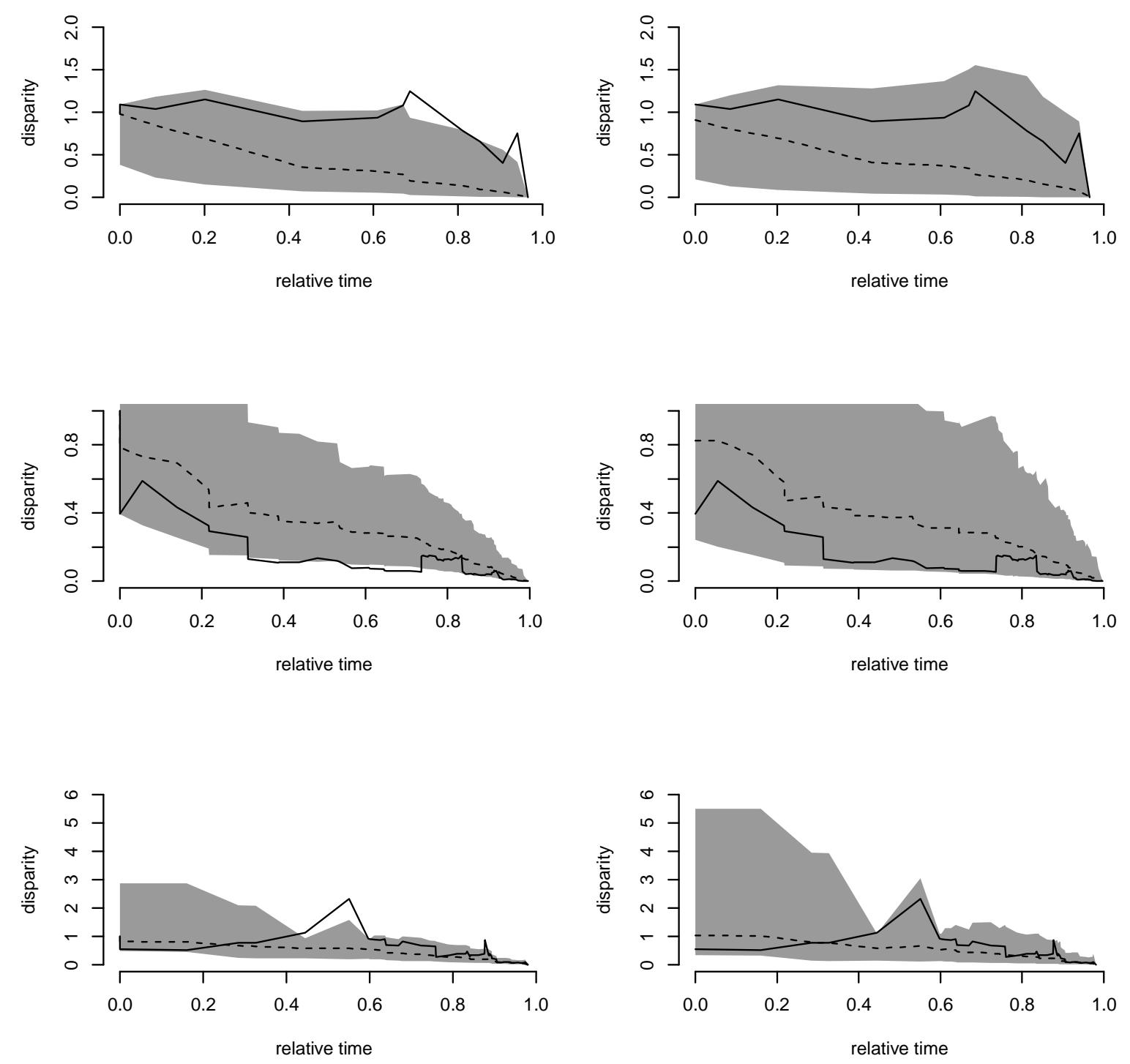

534 Figure 3. Comparisons of inference from using the pointwise envelope test (left hand column) and

535 the rank envelope test (right hand column) for trait disparity through time for three showcase

536 datasets. In each panel, the empirical pattern (solid black line) is compared to the median of 5000

537 simulations of the null model of Brownian evolution (broken lines), and the shaded regions

538 correspond to the $95 \%$ confidence intervals calculated using the pointwise (left column) and rank

539 envelope (right column) methods. Top row is for Darwin's finches (Geospiza) and the evolution of

540 culmen length; middle row is for the evolution of Cetacean body size (Slater et al., 2010); bottom

541 row is for the evolution of anal fin shape in 131 species of African cichlids (Feilich, 2016). 


\section{Supplementary Information}
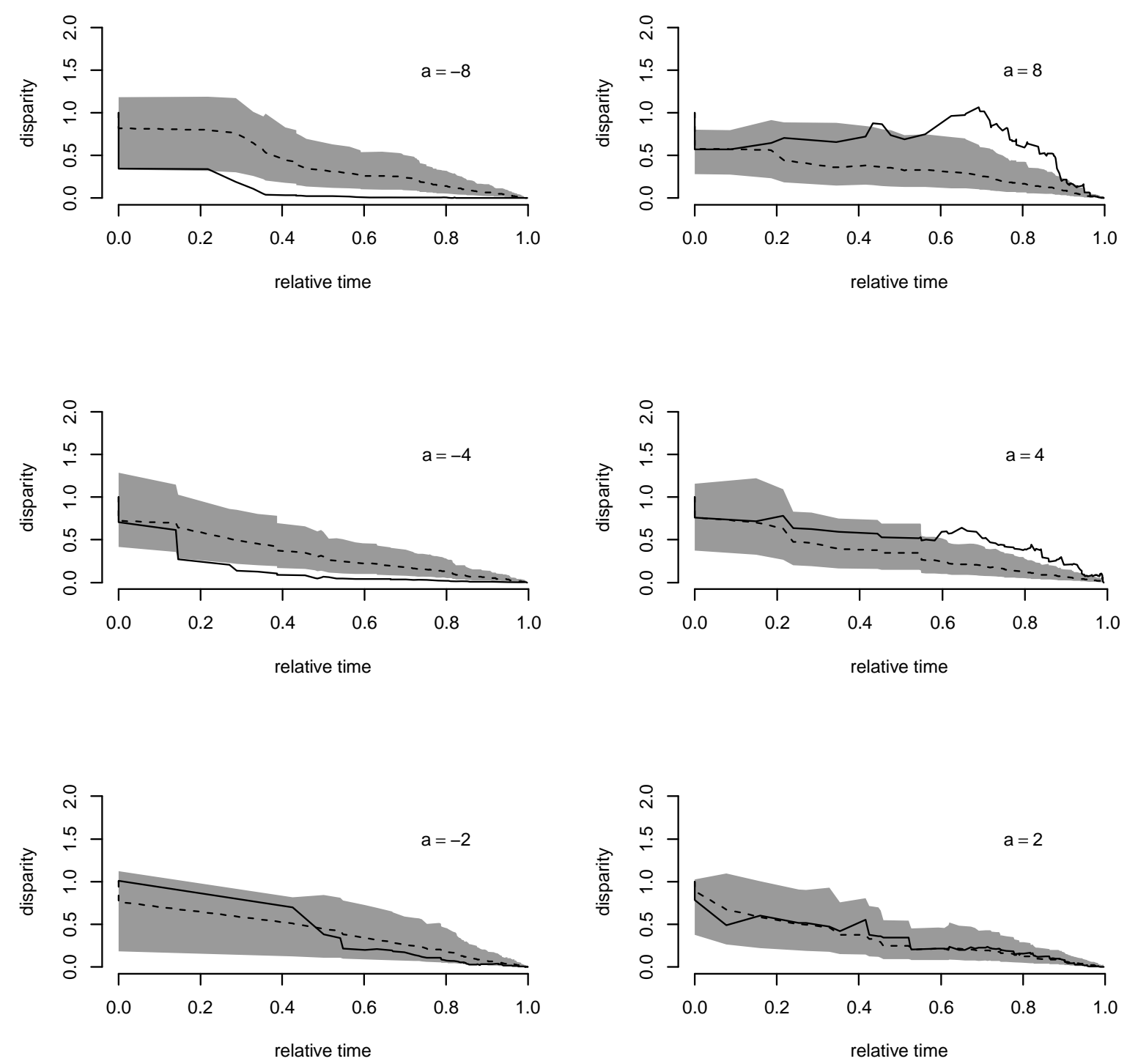

546 Figure S1. Examples of simulated data sets for disparity through time analyses where the rate of

547 trait evolution either slows down $(a<0)$ or speeds up $(a>0)$ over time (black solid lines). Broken

548 lines represent the median disparity through time for 2500 simulations of the null model of

549 Brownian evolution (ie $a=0$ ), and the shaded region is the $95 \%$ confidence interval for the

550 simulations based upon the rank envelope method (see main text). Simulations are for simulated

551 phylogenetic trees with 100 species at the tips. 

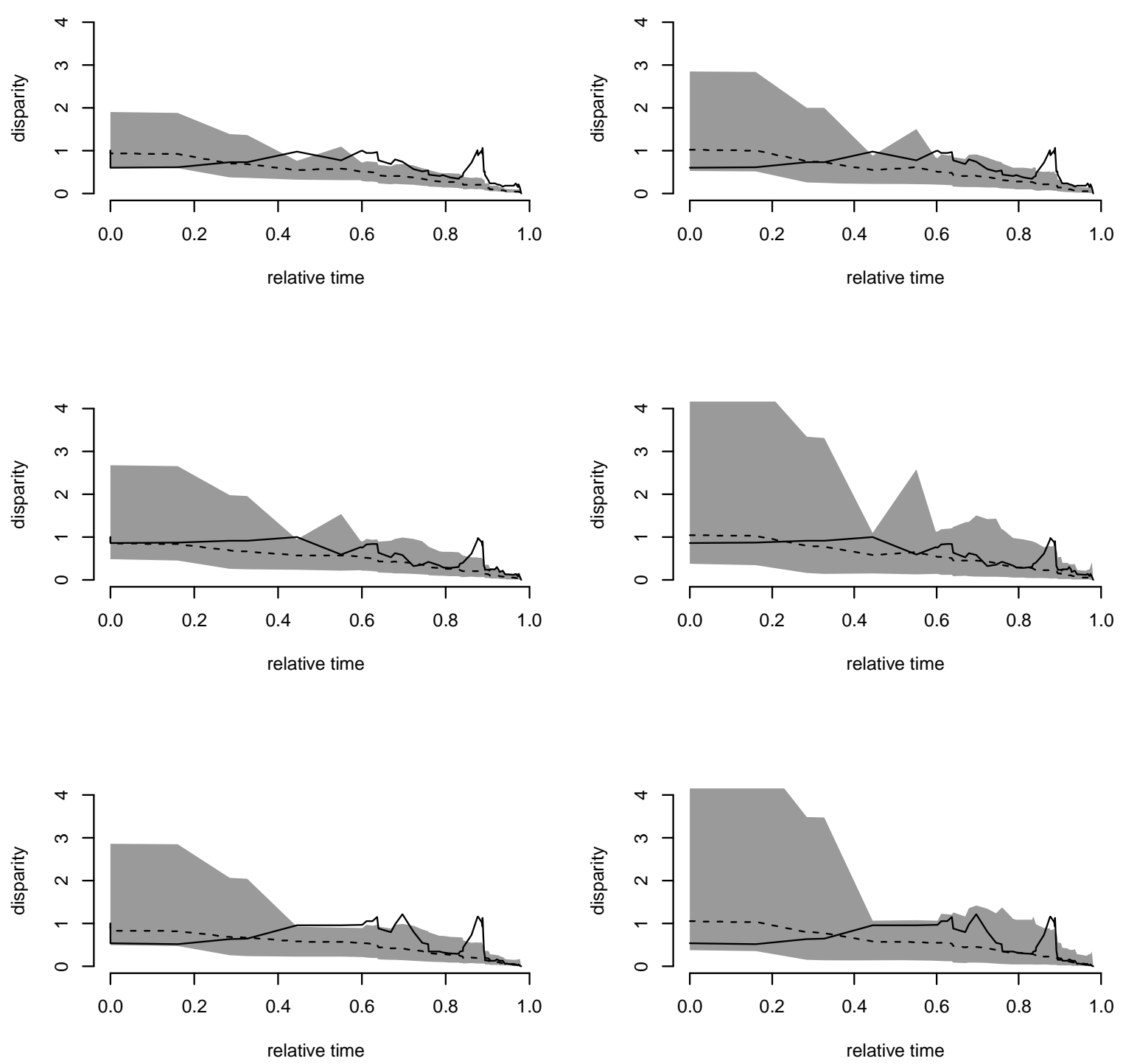

553

554 Figure S2. Comparisons of inference from using the (left hand column) pointwise envelope test and

555 the (right hand column) rank envelope test for trait disparity through time for (top row) body shape;

556 (middle row) caudal fin shape; and (bottom row) dorsal fin shape, for 131 species of African cichlid

557 fishes. Data is taken from (Feilich, 2016). In each panel the empirical pattern (solid black line) is

558 compared to the median of 5000 simulations of the null model of Brownian evolution of the trait

559 values (broken lines) on the phylogenetic tree, and the shaded regions correspond to the $95 \%$

560 confidence region. 